\title{
REFERENCES
}

1. Zygmund, Trigonometrical series, chap. 5, p. 123.

2. Ibid., chap. 2, p. 32 .

UNIVERSITY OF WISCONSIN

\section{ON FIBRE SPACES. II}

RALPH H. FOX

This paper is primarily concerned with fibre mappings ${ }^{1}$ into an absolute neighborhood retract. Theorem ${ }^{2} 3$ is a converse of the covering homotopy theorem; it characterizes fibre mappings (into a compact ANR) as mappings for which the covering homotopy theorem holds. Theorem 4 is Borsuk's fibre theorem; ${ }^{3}$ the proof ${ }^{4}$ which I present here is new. It seems to me that this theorem is a promising tool in function-space theory. Also I think that it furnishes conclusive justification for the generality of the Hurewicz-Steenrod definition of a fibre space. In fact, a fibre space of the type constructed by Borsuk's theorem almost never has a compact base space and almost never has its fibres of the same topological type.

The common denominator of the proofs of Theorems 3 and 4 is a property which I call local equiconnectivity. Local equiconnectivity is a strengthened form of local contractibility and a weakened form of the absolute neighborhood retract property (Theorems 1 and 2). Definitions and notations are those of FS. I. ${ }^{5}$

Let $\Delta$ be the diagonal subset $\sum_{b \in B}(b, b)$ of $B \times B$. I shall call the space $B$ locally equiconnected (or, to be specific, $(U, V)$-equiconnected) if there are neighborhoods $U$ and $V$ of $\Delta$ and a homotopy $\lambda$ in $B$ between the two projections of $U$ which does not move the points of $\Delta$ and which is uniform ${ }^{5}$ with respect to $V$. Precisely:

(1) $\lambda_{t}\left(b_{0}, b_{1}\right)$ is defined for all $\left(b_{0}, b_{1}\right) \in U$,

(2) $\lambda_{0}\left(b_{0}, b_{1}\right)=b_{0}$,

Received by the editors April 2, 1943.

${ }^{1}$ W. Hurewicz and N. Steenrod, Proc. Nat. Acad. Sci. U. S. A. vol, 27 (1941) p. 61.

2 This theorem was announced in Hurewicz-Steenrod, op. cit. footnote 3.

${ }^{3}$ K. Borsuk, Fund. Math. vol. 28 (1937) p. 99.

4 This proof was announced in the author's paper On the deformation retraction of some function spaces $\cdots$, Ann. of Math. vol. 44 (1943) p. 52.

$5 \bar{\pi}(x, b)=(\pi(x), b)$ as in R. H. Fox, On fibre spaces. I, Bull. Amer. Math. Soc. vol. 49 (1943) pp. 555-557. 
(3) $\lambda_{1}\left(b_{0}, b_{1}\right)=b_{1}$

(4) $\lambda_{t}(b, b)=b$ for every $(b, b) \in \Delta, 0 \leqq t \leqq 1$,

(5) there is a $\delta>0$ such that $\left|t-t^{\prime}\right|<\delta$ implies that $\sum_{\left(b_{0}, b_{1}\right) \in U}\left(\lambda_{t}\left(b_{0}, b_{1}\right), \lambda_{t^{\prime}}\left(b_{0}, b_{1}\right)\right) \subset V$.

Roughly speaking, $B$ is locally equiconnected if there are paths between sufficiently nearby points such that the paths depend continuously on the end points.

THEOREM 1. A locally equiconnected space is locally contractible.

Let $N$ be a neighborhood of some point $b_{1}$ of $B$ and let $M$ denote the set of points $b_{0}$ such that $\sum_{0 \leqq t \leqq 1} \lambda_{t}\left(b_{0}, b_{1}\right) \subset N$. By (4), $b_{1} \in M$; a simple continuity argument shows that $M$ is a neighborhood of $b_{1}$. Since $M$ is contractible to $b_{1}$ in $N$ the theorem is proved.

Theorem 2. A compact $A N R$-set is locally equiconnected.

Let $B$ be a neighborhood retract of the Hilbert parallelotope $Q$ and let $r$ be a retraction of an open neighborhood $N$ of $B$ onto $B$. Since $Q-N$ and $B$ are disjoint compact sets $\epsilon=d(B, Q-N) / 2>0$. Let $U_{\epsilon}$ be the closed neighborhood of $\Delta$ determined by the covering of $B$ by $\epsilon$-spheres and let $\lambda_{t}\left(b_{0}, b_{1}\right)=r\left((1-t) b_{0}+t b_{1}\right)$ for $\left(b_{0}, b_{1}\right) \in U_{\epsilon}$, $0 \leqq t \leqq 1$. Conditions (1), (2), (3), and (4) are obviously satisfied. Condition (5) follows, for any $V$, from the compactness of $U_{\epsilon}$.

From Theorems 1 and 2 it follows, ${ }^{6}$ for finite dimensional compacta, that local contractibility, local equiconnectivity and the ANR property are equivalent. For infinite dimensional spaces no more is known than is implied above.

THEOREM 3 (CONVERSE OF THE COVERING HOMOTOPY THEOREM). Let $B$ be a $(U, V)$-equiconnected space and let $\pi \in B^{X}$. Suppose that for every mapping $g \in X^{Y}$ and homotopy $h$ in $B$ which is uniform with respect to $V$ and has initial value ${ }^{5} \pi$ there exists a covering homotopy $h^{*}$ in $X$ with initial value $g$. Then $\pi$ is a fibre mapping relative to $U$.

Let $h_{t}(x, b)=\lambda_{t}(\pi(x), b)$. Since $h$ is uniform with respect to $V$ there is a covering homotopy $h^{*}$ such that $h_{0}^{*}(x, b)=x$. Let $\phi(x, b)=h_{1}^{*}(x, b)$. Then ${ }^{5} \phi$ maps $\bar{\pi}^{-1}(U)$ continuously into $X$ and $\pi \phi(x, b)=b$. Since $h_{[0,1]}(x, \pi(x))=\pi(x)$ it follows that $\phi(x, \pi(x))=h_{1}^{*}(x, \pi(x))=h_{0}^{*}(x, \pi(x))$ $=x$. Thus $\phi$ is a slicing function.

Let $A$ be a closed subset of $X$ and let $\pi$ denote the sectioning operation $\pi(f)=f \mid A, f \in Y^{x}$.

${ }^{6}$ K. Borsuk, Fund. Math. vol. 19 (1932) p. 240, Theorem 32. 
THEOREM 4 (BoRsuK's FIBRE THEOREM). If $A$ is closed in $X$ and $Y$ is a compact $A N R$-set then $\pi$ is a fibre mapping.

By Theorem 2, $Y$ is locally equiconnected and, if it is suitably metrized, there is a positive number $\epsilon$ such that $\lambda_{t}\left(y_{0}, y_{1}\right)$ is defined whenever $d\left(y_{0}, y_{1}\right)<\epsilon$. Let $\Gamma_{0}$ denote the graph of $\pi$ and let $\Gamma_{\epsilon}$ denote the subset of $Y^{x} \times Y^{A}$ defined by the rule $(f, g) \in \Gamma_{\epsilon}$ when $d(\pi(f), g)<\epsilon$. Because $Y$ is compact $\Gamma_{\epsilon}$ is a neighborhood of $\Gamma_{0}$. Define

$$
\psi_{t}(f, g, x)= \begin{cases}\lambda_{t}(f(x), g(x)) & \text { for } \quad(f, g, x) \in \Gamma_{\epsilon} \times A, \\ f(x) & \text { for } \quad(f, g, x) \in \Gamma_{0} \times X .\end{cases}
$$

Thus $\psi$ is a homotopy in $Y$; each $\psi_{t}$ is defined on the closed subset $C=\Gamma_{\epsilon} \times A+\Gamma_{0} \times X$ of $\Gamma_{\epsilon} \times A$. But $\psi_{0}(f, g, x)=f(x)$ for every $(f, g, x)$ $\in C$, and this map has the extension $\psi_{0}^{*}(f, g, x)=f(x)$ defined for every $(f, g, x) \in \Gamma_{\epsilon} \times X$. It follows ${ }^{7}$ that $\psi_{1}$ can be extended to $\Gamma_{\epsilon} \times X$. Let $\psi_{1}^{*}$ denote an extension of $\psi_{1}$ and set $\phi(f, g)(x)=\psi_{1}^{*}(f, g, x)$ for $(f, g) \in \Gamma_{\epsilon}$ and $x \in X$, so that $\phi(f, g) \in Y^{x}$ for every fixed $(f, g) \in \Gamma_{\epsilon}$. Then $\phi$ maps $\Gamma_{\epsilon}$ into $Y^{X}, \pi \phi(f, g)=g, \phi(f, \pi(f))=f$. Thus $\phi$ is a slicing function for $\pi$.

Since the image set of a fibre mapping is necessarily open and closed in the base space, an example " $\varepsilon$ " shows that Theorem 4 is false for non-compact ANR-sets $Y$. However if neither $X$ nor $Y$ are compact (as in " $\mathcal{~ " ) ~ t h e ~ t o p o l o g y ~ o f ~} Y^{X}$ (and also of $Y^{A}$ ) depends on the metrization of $Y$. Thus it may be possible (as it is in " $\varepsilon$ ") to remetrize an ANR-set $Y$ so as to make the sectioning operations fibre mappings. It should be observed that Borsuk has shown that Theorem 4 is false (with or without remetrization) if $Y$ is not locally contractible. ${ }^{4}$

UNIVERSITY OF ILLINOIS

${ }^{7}$ W. Hurewicz and H. Wallman, Dimension theory, Princeton, 1941, p. 86.

${ }^{8}$ R. H. Fox, Bull. Amer. Math. Soc. vol. 48 (1942) p. 271 footnote 3. 